\title{
New General Approach to Airplane Rotation Analysis
}

\author{
By Milan Bajovic, ${ }^{1)}$ Milovan Zivanovic, ${ }^{2)}$ Bosko Rasuo ${ }^{3)}$ and Predrag Stojakovic ${ }^{1)}$ \\ ${ }^{1)}$ Aeronautical Institute, Zarkovo, Belgrade, Serbia \\ ${ }^{2)}$ Mihailo Pupin Institute, Belgrade, Serbia \\ ${ }^{3)}$ Faculty of Mechanical Engineering, University of Belgrade, Belgrade, Serbia
}

(Received May 27th, 2009)

\begin{abstract}
The paper presents a new approach to analysis of dynamic properties of the rotating airplane. The analysis is based on the rate of rotational energy and a full non-linear model of airplane dynamics. It can be applied to a whole range of attack angles. To provide stable rotation, it is sufficient to use algebra and know only the inertial properties of the airplane and the functional relations of either aerodynamic moments or angular accelerations. We prove that the results are fully congruent with those obtained by conventional methods within the scope of validity of the simplified model.
\end{abstract}

Key Words: Airplane Rotation, Flight Dynamics, Stability, High Angles of Attack, Non-linear Model

\section{Introduction}

Adequate assessment of airplane behavior in performing all modes of rotational motion is a crucial problem in designing the dynamic properties of a fixed-wing aircraft. Design tools available at airplane construction have governed the approach to this problem and, historically, the first examples were separate analysis of linearized pitching and rolling motion using model of each motion mode with limited scope of validity. This approach belongs to the "bottom-up" category of design procedures, but cannot predict airplane behavior in stall, deep-stall and spin flight regimes. The best known example is Blakelock. ${ }^{1)}$

The general designer's approach is governed by the need to obtain the required airplane dynamics in the predefined flight regime. The appearance of requirements for increased scope of controlled airplane maneuvers has been balanced by simultaneous improvement of design tools in the form of adequate mathematical models of dynamics implemented in an adequate computational environment. The requirements of airplane maneuvers demand evaluation of airplane dynamics with the pilot-in-the-loop in the design phase. This "top-down" approach resulted in a set of practical mathematical models of airplane dynamics comprising a full range of stream variables and angular velocities, with the advantage that the quality of solutions depends only on the quality of input data. The most notable example is Etkin. ${ }^{2)}$

Furthermore, the generated model of airplane dynamics serves as the kernel for the model of closed-loop aircraft dynamics, with power plant and flight control system (FCS) models and real or modeled pilot as the main supplementary subsystems. Research in this domain has been conducted in three directions. The first has been aimed at experimental determination of aerodynamic shapes that enable flight control in a widest possible airplane state space, presented in reports by Ref. 3) and Ref. 4). In the

C 2010 The Japan Society for Aeronautical and Space Sciences second, results have been sought based on theory of catastrophes, ${ }^{5)}$ and the theory of bifurcations. $\left.{ }^{6}\right)$ The third, has been flight simulation in deep stall and spin, with input parameters obtained from the first direction. This enabled analysis of the dynamics of the airplane with an open and closed control loop. ${ }^{78)}$ The last method is used most widely because hardware-in-the-loop simulation within the whole range of flight regimes is mandatory during airplane-FCS integration. A common feature of all three approaches is that the conclusion about the characteristics of airplane dynamics is reached on the basis of analysis of the elements from the set of results obtained.

The main disadvantage of the previous methods is that conclusions in the design process are drawn 'a-posteriori', after the shape and/or inertial properties of the airplane have already been determined, or, they validate the already defined aeroplane parameters. What the designer really needs to know is how to define the shape and/or inertial properties of the airplane in order to obtain the required quality of airplane rotational motion. The 'designer's' method for the analysis of airplane rotation must first provide an integral analysis of airplane rotation within the whole range of stream angles and angular velocities, where partial (linear) models represent only special cases. Second, it must possess an 'a-priori' quality that enables definition of the shape and/or inertial properties of the airplane on the basis of the required qualities of airplane rotational motion. Finally, it must provide answers about the dynamics of airplane rotation at all stages of design, from the conceptual determination of airplane shape to the final stages of hardware-in-the-loop simulation and in-flight testing.

The basis of the method in this paper is observation of total airplane motion as a complex consisting of translational motion of airplane center of inertia and relative airplane rotational motion about this center of inertia. Kinetic energies of these two modes of motion are not mutually dependent. Therefore, kinetic energy of rotation is inde- 
pendently observed to define its rate and conditions to increase it, keeping it constant or decrease it. These conditions are related to the angle between vectors of angular velocity rate and gradient of the airplane rotational kinetic energy. The next step is to define the correlation between aerodynamic moment, angular velocity and rate of rotational kinetic energy. Variations in the translational motion of the airplane are due to the variations of the aerodynamic forces and moments. Variations of the aerodynamic forces and moments are caused by the airplane rotation relative to the natural flight path trihedron of the airplane center of inertia, the $X$-axis of this trihedron being along airplane velocity. The velocity of airplane rotation around the center of inertia in the inertial space is an order of magnitude greater than the velocity of rotation of the natural flight path trihedron of the airplane center of inertia. Therefore, physically the variations of the aerodynamic forces and moments are more related to the rotation of the airplane than to the rotation of the natural flight path trihedron of the airplane center of inertia. This justifies close correlation between aerodynamic moment, angular velocity, and rate of rotational kinetic energy.

\section{Theoretical Approach}

In developing the theoretical grounds for the rotation analysis in this paper, we assumed that the airplane is a rigid body, i.e. in the period of observation, its inertial properties are invariant with time. The overall non-linear mathematical model of airplane dynamics is obtained from the law of continuity of momentum and moment of momentum in the form

$$
\begin{aligned}
& \boldsymbol{F}=\boldsymbol{F}_{\mathrm{a}}+\boldsymbol{F}_{\mathrm{g}}+\boldsymbol{F}_{\mathrm{p}}=\frac{\partial(m \boldsymbol{V})}{\partial t}+\omega \times m \boldsymbol{V} \\
& \mathfrak{M}=\mathfrak{M}_{\mathrm{a}}+\mathfrak{M}_{\mathrm{p}}=\frac{\partial(\mathfrak{J} \omega)}{\partial t}+\omega \times \mathfrak{J} \omega
\end{aligned}
$$

where $\boldsymbol{F}$ is the vector of resulting force, $\boldsymbol{F}_{\mathrm{a}}, \boldsymbol{F}_{\mathrm{g}}, \boldsymbol{F}_{\mathrm{p}}$ are, respectively, the vectors of resulting aerodynamic, gravitational and propulsive force, $\mathfrak{M}$ is the resulting moment of force, $\mathfrak{M}_{\mathrm{a}}=\operatorname{col}\left(M_{\mathrm{a}}, L_{\mathrm{a}}, N_{\mathrm{a}}\right), \mathfrak{M}_{\mathrm{p}}$ are the vectors of resulting moments of aerodynamic forces and propulsive group, $m$ is the mass and $\mathfrak{J}$ is the general form of inherently symmetric tensor of inertia of the airplane, $\boldsymbol{V}=\operatorname{col}(u, v, w)$ and $\omega=\operatorname{col}(p, q, r)$ are the vectors of airplane translational and angular velocities. Variables $L_{\mathrm{a}}, M_{\mathrm{a}}, N_{\mathrm{a}} ; u, v, w$ and $p, q, r$ are, respective projections of the vectors $\mathfrak{M}_{\mathrm{a}}, \boldsymbol{V}$ and $\omega$ on the $x, y, z$ axes of the airplane body coordinate system. By solving Eq. (1) on highest-derivatives, the following set of equations is obtained:

$$
\begin{aligned}
\dot{\boldsymbol{V}} & =\frac{1}{m}\left[\boldsymbol{F}_{\mathrm{a}}+\boldsymbol{F}_{\mathrm{g}}+\boldsymbol{F}_{\mathrm{p}}-(\omega \times m \boldsymbol{V})\right] \\
\dot{\omega} & =\mathfrak{J}^{-1}\left[\mathfrak{M}_{\mathrm{a}}+\mathfrak{M}_{\mathrm{p}}-(\omega \times \mathfrak{J} \omega)\right] .
\end{aligned}
$$

The dot over the variable denotes its rate, i.e. first-order time derivative. The kinetic energy of rotation $E_{\mathrm{R}}=$ $1 / 2 \omega^{\mathrm{T}} \mathfrak{J} \omega$ of the airplane with the plane of inertial symmetry $0 x z$, or, briefly, rotational energy, is of the inherent form

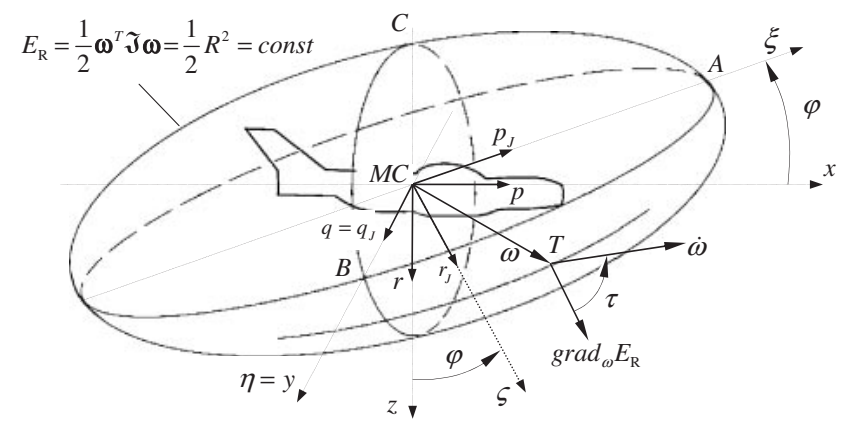

Fig. 1. Ellipsoid of rotation.

$$
\begin{aligned}
2 E_{\mathrm{R}} & =\omega^{\mathrm{T}} \mathfrak{J} \omega \\
& =I_{x} p^{2}+I_{y} q^{2}+I_{z} r^{2}-2 I_{x z} p r \\
& =J_{1} p_{J}^{2}+J_{2} q_{J}^{2}+J_{3} r_{J}^{2} \\
& =R^{2}
\end{aligned}
$$

where $I_{x}, I_{y}, I_{z}$ are the axial and $I_{x z}$ centrifugal moments of inertia about the corresponding axes of the airplane body coordinate system. If the fuel sloshing, control surface movement and other similar phenomena can be neglected, the tensor of inertia $\mathfrak{J}$ is invariant with time. Scalar $R^{2}>0$ is defined as the intensity of rotation, the square of its value being two times the amount of rotational energy. Equation (3) represents the equation of ellipsoid of rotational energy or, briefly, ellipsoid of rotation (Fig. 1). The three main orthogonal axes of the ellipsoid of inertia $(\xi, \eta, \zeta)$ are collinear with the three main axes of inertia of the airplane and intersect at the airplane center of mass. The angular velocity presented through the components in this system is $\omega=\operatorname{col}\left(p_{J}, q_{J}, r_{J}\right)$, and the tensor of inertia has the form $\operatorname{diag}\left(J_{1}, J_{2}, J_{3}\right), J_{1}, J_{2}, J_{3}$ being the main moments of inertia. For an inertially symmetric airplane, the ellipsoid of rotation is turned about the $y$-axis of airplane body coordinate system by the angle $\Phi=$ $1 / 2 \cdot \arctan \left[2 I_{x z} /\left(I_{x}-I_{z}\right)\right]$. Values of the main half-axes of the ellipsoid of rotation (point $A, B, C$ ) are $\lambda_{1}=R / \sqrt{J_{1}}$, $\lambda_{2}=R / \sqrt{J_{2}}$ and $\lambda_{3}=R / \sqrt{J_{3}}$. During maneuvering flight, the ellipsoid of rotation varies with time, i.e. $R=R(t)$. In the absence of rotation it becomes a point. With an increase/decrease of rotation intensity, the ellipsoid of rotation inflates or deflates. The origin of the vector of angular velocity $\omega$ is at the intersection of the main axes of inertia, while its apex is touching the surface of the ellipsoid of rotation at point $T$. The normal to the surface of the ellipsoid of rotation at $T$ is $\vec{n}=c \cdot \operatorname{grad}_{\omega} E_{\mathrm{R}}, c \in R^{1}$, i.e. the gradient of energy of rotation along the vector of angular velocity $\omega$.

The vector of the rate of angular velocity, $\dot{\omega}$, has the origin at $T$. The kinetic energy of rotation $E_{\mathrm{R}}$ is a scalar function of $\omega$, therefore, the rate of change of $E_{\mathrm{R}}$ is

$$
\frac{\mathrm{d}\left(E_{\mathrm{R}}\right)}{\mathrm{d} t}=\dot{E}_{\mathrm{R}}=\left[\operatorname{grad}_{\omega} E_{\mathrm{R}}\right]^{\mathrm{T}} \cdot \dot{\omega}
$$

i.e. its rate along the angular velocity vector $\omega$. To simplify geometric presentation, let us denote as $\tau$ the relative angle 
between $\operatorname{grad}_{\omega} E_{\mathrm{R}}$ and the rate of angular velocity $\dot{\omega}$ (Fig. 1). When the intensity of rotation $R$ is constant, the vector of the rate of angular velocity $\dot{\omega}$ is a tangent to the ellipsoid of rotation. Then, $\tau$ is a right angle, making the inner product $\left[\operatorname{grad}_{\omega} E_{\mathrm{R}}\right]^{\mathrm{T}} \cdot \dot{\omega}=0$. When the intensity of rotation is increasing, $\tau$ is becoming acute, with the inner product $\left[\operatorname{grad}_{\omega} E_{\mathrm{R}}\right]^{\mathrm{T}} \cdot \dot{\omega}>0$ and the airplane rotational motion diverges. In contrast, when the intensity of rotation is decreasing, $\tau$ is becoming obtuse, with the inner product $\left[\operatorname{grad}_{\omega} E_{\mathrm{R}}\right]^{\mathrm{T}} \cdot \dot{\omega}<0$ and the airplane rotational motion converges. Therefore, to prevent the increase of rotation, the condition $\left[\operatorname{grad}_{\omega} E_{\mathrm{R}}\right]^{\mathrm{T}} \cdot \dot{\omega}=0$ must be satisfied. However, if it is to be stopped, $\left[\operatorname{grad}_{\omega} E_{\mathrm{R}}\right]^{\mathrm{T}} \cdot \dot{\omega}<0$ must be fulfilled. Notice that the airplane control intentionally generates the condition $\left[\operatorname{grad}_{\omega} E_{\mathrm{R}}\right]^{\mathrm{T}} \cdot \dot{\omega}>0$.

The next step is to correlate this condition to aerodynamic moments. Initially, it is established that

$$
\begin{aligned}
\operatorname{grad}_{\omega} E_{\mathrm{R}} & =\operatorname{grad}_{\omega}\left(\frac{1}{2} \omega^{\mathrm{T}} \mathfrak{J} \omega\right) \\
& =\mathfrak{J} \omega=\omega^{\mathrm{T}} \mathfrak{J} .
\end{aligned}
$$

The vector of the rate of angular velocity $\dot{\omega}$ is defined from the second equation in (2). The moment of propulsive group has two parts. The first, $\mathfrak{M}_{\mathrm{p}}^{\mathrm{P}}$, is generated by the propulsive force of the engine(s), while the second, $\mathfrak{M}_{\mathrm{p}}^{\mathrm{G}}$, is generated by gyroscopic moments, so

$$
\begin{aligned}
\mathfrak{M}_{\mathrm{p}}= & \mathfrak{M}_{\mathrm{p}}^{\mathrm{P}}+\mathfrak{M}_{\mathrm{p}}^{\mathrm{G}} \\
= & \sum_{i}\left(\boldsymbol{r}_{i} \times \boldsymbol{F}_{\mathrm{p} i}\right)+\sum_{j}\left(I_{\mathrm{p} j}\left(\omega_{\mathrm{p} j} \times \omega\right)\right) ; \\
& \quad i=1, \ldots, n, \quad j=1, \ldots, k
\end{aligned}
$$

In Eq. (6), $n$ is the total number of propulsors on the airplane and $k$ is the total number of rotating elements in these propulsors that generate significant gyroscopic moments. The scalar product of a vector with a vector product when one of the multipliers is that vector, is zero; hence, when multiplied by $\omega^{\mathrm{T}} \mathfrak{J}$, the elements from Eq. (2) become $\omega^{\mathrm{T}}(\omega \times \mathfrak{J} \omega)=0$ and $\omega^{\mathrm{T}} \mathfrak{M}_{\mathrm{p}}^{\mathrm{G}}=\omega^{\mathrm{T}}\left(I_{\mathrm{p}}\left(\omega_{\mathrm{p}} \times \omega\right)\right)=0$. If the active moments generated by aerodynamic and propulsive forces are denoted $\mathfrak{M}^{\mathrm{A}}$, then

$$
\begin{aligned}
\mathfrak{M} & =\mathfrak{M}^{\mathrm{A}}=\mathfrak{M}_{\mathrm{a}}+\mathfrak{M}_{\mathrm{p}}^{\mathrm{P}} \\
& =\left[\begin{array}{c}
L_{\mathrm{a}}+L_{\mathrm{p}}^{\mathrm{P}} \\
M_{\mathrm{a}}+M_{\mathrm{p}}^{\mathrm{P}} \\
N_{\mathrm{a}}+N_{\mathrm{p}}^{\mathrm{P}}
\end{array}\right]=\left[\begin{array}{c}
L \\
M \\
N
\end{array}\right] .
\end{aligned}
$$

Consequently,

$$
\begin{aligned}
\dot{E}_{\mathrm{R}} & =\left[\operatorname{grad}_{\omega} E_{\mathrm{R}}\right]^{\mathrm{T}} \dot{\omega} \\
& =\omega^{\mathrm{T}} \mathfrak{J} \dot{\omega}=\omega^{\mathrm{T}}\left(\mathfrak{M}_{\mathrm{a}}+\mathfrak{M}_{\mathrm{p}}^{\mathrm{P}}\right) \\
& =\omega^{\mathrm{T}} \mathfrak{M}^{\mathrm{A}}=L p+M q+N r .
\end{aligned}
$$

Therefore, the scalar equation defining the condition for preventing increase and for stoppage of the airplane rotation, i.e. non-divergent airplane rotation, is

$$
\begin{aligned}
& \dot{E}_{\mathrm{R}}=\left[\operatorname{grad}_{\omega} E_{\mathrm{R}}\right]^{\mathrm{T}} \dot{\omega}=\omega^{\mathrm{T}} \mathfrak{J} \dot{\omega} \leq 0 \\
& \dot{E}_{\mathrm{R}}=\mathfrak{M}^{\mathrm{T}} \omega=L p+M q+N r \leq 0
\end{aligned}
$$

These general conditions have been obtained without any simplification in the description of airplane dynamics. They stem from the inherent form of kinetic energy of rotation in Eq. (3). The physical meaning of Eqs. (9) and (10) is simple. As $\dot{E}_{\mathrm{R}}=\mathfrak{M}^{\mathrm{T}} \omega$ represents the necessary power to change the energy of rotation, the positive power increases energy of rotation, while the negative decreases it. The condition in Eq. (9) correlates angular velocity, acceleration and inertial properties of the airplane. As the tensor of inertia $\mathfrak{J}$ is considered invariant of time, condition (9) correlates angular velocity and its rate. The condition (9) can then be readily used to define control laws that will prevent divergence of rotational motion of the airplane. When the angular velocity and its rate are measured in flight, Eq. (9) univocally defines the rate of rotational kinetic energy and its sign, whereas Eq. (3) can be used to define rotational energy and ellipsoid of rotation. When the components of airplane active moment are known within the airplane state space, either as the total values, or as the functions of vectors of airplane state and input, the condition in Eq. (10) can be used to correlate components of angular velocity and moments acting on the airplane. Therefore, the condition in Eq. (10) is suitable for use during design of the airplane, because it can be used to define the boundaries within which it is possible to prevent divergence of rotation. The condition in Eq. (10) is analyzed later.

Equation (10) contains in its active moments all controls available to the airplane during flight along a flight path, the aerodynamic $\left(\delta_{l}, \delta_{m}, \delta_{n}\right)$ and propulsive $\left(\delta_{\mathrm{T}}\right)$ ones. Since this paper focuses on the airplane design procedure, further simplification is made by assuming that the resulting thrust force $\vec{F}_{\mathrm{p}}=\vec{T}_{\mathrm{p}}$ is acting through the airplane center of inertia, so that $\mathfrak{M}_{\mathrm{p}}^{\mathrm{P}}=0$. Then, the condition in Eq. (10) reduces to

$$
\dot{E}_{\mathrm{R}}=\mathfrak{M}_{\mathrm{a}}^{\mathrm{T}} w=L_{\mathrm{a}} p+M_{\mathrm{a}} q+N_{\mathrm{a}} r \leq 0 .
$$

To assess the capabilities of the control of airplane rotation by aerodynamic moments only, it is necessary to know the functional dependence of its components $L_{\mathrm{a}}, M_{\mathrm{a}}, N_{\mathrm{a}}$. The aerodynamic moment $\mathfrak{M}_{\mathrm{a}}=\operatorname{col}\left(L_{\mathrm{a}}, M_{\mathrm{a}}, N_{\mathrm{a}}\right)$ is a nonlinear function of airplane state variables and control surface deflection, as well as of the rate of both, or

$$
\mathfrak{M}_{\mathrm{a}}=\mathfrak{M}_{\mathrm{a}}(\boldsymbol{X}, \dot{\boldsymbol{X}}, \boldsymbol{U}, \dot{\boldsymbol{U}}) .
$$

Translatory components are defined and measured in the wind, or stream axis coordinate system; for the rotation the same is done in the body axis system. The airplane state vector is then $\boldsymbol{X}=\operatorname{col}\left(V, \beta, \alpha, p, q, r, \Phi, \Theta, \Psi, x_{0}, y_{0}, z_{0}\right)$, where $V$ is the intensity of velocity, $\alpha, \beta$ are the angles of sideslip and attack, $\Phi, \Theta, \Psi$ are Euler angles, and $x_{0}, y_{0}, z_{0}$ are the Earth fixed coordinates. The control vector $\boldsymbol{U}=\operatorname{col}\left(\delta_{l}, \delta_{m}, \delta_{n}\right)$ contains deflections of roll (ailerons), pitch (elevator), and yaw (rudder) commands. For atmospheric flight, there is a biunivocal correspondence between the airplane state vector $\boldsymbol{X}$ and the stream parameters, Mach 
and Reynolds numbers. Then, with the stream parameters known, the functional relation for the vector of aerodynamic moment, with precision of up to the first-order time derivatives, is of the form

$$
\mathfrak{M}_{\mathrm{a}}=\mathfrak{M}_{\mathrm{a}}\left(\alpha, \dot{\alpha}, \beta, \dot{\beta}, p, q, r, \delta_{l}, \dot{\delta}_{l}, \delta_{m}, \dot{\delta}_{m}, \delta_{n}, \dot{\delta}_{n}\right) .
$$

An important aspect in the presentation of aerodynamic moment is that, in most cases, it can be modeled as being additive in regard to the control vector $\boldsymbol{U}$, i.e. it consists of the 'reference' $\mathfrak{M}_{\mathrm{a}}^{\mathrm{r}}$ and 'control' $\mathfrak{M}_{\mathrm{a}}^{\mathrm{c}}$ part. Within the airplane state space they can be presented by either total values or functional relations, or

$$
\begin{aligned}
\mathfrak{M}_{\mathrm{a}} & =\mathfrak{M}_{\mathrm{a}}^{\mathrm{r}}+\mathfrak{M}_{\mathrm{a}}^{\mathrm{c}} \\
& =\mathfrak{M}_{\mathrm{a}}^{\mathrm{r}}(\boldsymbol{X}, \dot{\boldsymbol{X}})+\mathfrak{M}_{\mathrm{a}}^{\mathrm{c}}(\boldsymbol{X}, \dot{\boldsymbol{X}}, \boldsymbol{U}, \dot{\boldsymbol{U}}) \\
& =\left[\begin{array}{c}
L_{\mathrm{a}}^{\mathrm{r}}+L_{\mathrm{a}}^{\mathrm{c}} \\
M_{\mathrm{a}}^{\mathrm{r}}+M_{\mathrm{a}}^{\mathrm{c}} \\
N_{\mathrm{a}}^{\mathrm{r}}+N_{\mathrm{a}}^{\mathrm{c}}
\end{array}\right] .
\end{aligned}
$$

At the airplane design, the easiest and most precise method to determine functional dependences of aerodynamic moments is obtained by measurements in wind tunnels.

It is important to note that Eq. (10) is an exact analytical expression, regardless of how the components of aerodynamic moment, $L_{\mathrm{a}}, M_{\mathrm{a}}, N_{\mathrm{a}}$ are obtained. Equation (10) is the condition derived for an arbitrary time instant that is valid regardless of the history of the airplane motion. It is obtained without using analytical methods based upon the differential equations of motion. The exactness of analytical approach to the dynamics of airplane rotation based on Eq. (10) is constrained only by the exactness of the method of obtaining components of the aerodynamic moment $\mathfrak{M}_{\mathrm{a}}$.

The significance of Eq. (10) is its applicability. It is valid throughout the whole range of airplane state space, and can be used to assess properties of airplane rotation dynamics, providing a convenient analytical tool. Furthermore, whenever the aerodynamic moment $\mathfrak{M}_{\mathrm{a}}$ can be modeled as in Eq. (14), Eq. (10) can be rendered as

$$
\begin{aligned}
\dot{E}_{\mathrm{R}} & =\mathfrak{M}_{\mathrm{a}}^{\mathrm{T}} w \\
& =\left(L_{\mathrm{a}}^{\mathrm{r}}+L_{\mathrm{a}}^{\mathrm{c}}\right) p+\left(M_{\mathrm{a}}^{\mathrm{r}}+M_{\mathrm{a}}^{\mathrm{c}}\right) q+\left(N_{\mathrm{a}}^{\mathrm{r}}+N_{\mathrm{a}}^{\mathrm{c}}\right) r \leq 0 .
\end{aligned}
$$

Because Eq. (15) allows separate observation of the influence of airplane control, it can be readily used as a design tool, applicable $a b$ initio in designing the desired properties of airplane rotation dynamics.

\section{Locally Linearized Model of Aerodynamic Moment}

In the previous discussion, the general form of the condition for non-divergent rotation of the airplane Eq. (10) has been transformed into the reduced form (Eq. (15)) suitable for design of desirable properties of airplane rotation dynamics. The classical methods of analysis of airplane rotation are based on models of separated longitudinal and lateral-directional motion, valid within the limited domain of airplane state and input vectors. The aim of this paper is to prove that within the limited domain of validity of these models, analysis of airplane rotation based on the condition in Eq. (10) is fully congruent with analysis based on these separated models. Then, the results of analysis by classical methods are only particular solutions of the general condition stemming from Eq. (10) and this general condition enables analysis of airplane rotation outside the above areas of congruency.

The model of aerodynamic moments given by the functional relation Eq. (14) can be linearized locally throughout the whole range of state and space vectors with the domain of validity defined by the required exactness. Within the set of linearized models of aerodynamic moment, the locally linearized one is characterized by highest complexity. It preserves validity in a sufficiently small vicinity of any point within the range of stream angles, where effects of nonstationary flow field and cross-influence can be neglected. Then, the elements of first-order and higher derivatives can be neglected and the model of aerodynamic moment can be derived with precision of up to the zero-order derivatives. Let the linearized model of aerodynamic moment be derived in the vicinity of reference values of the angle of attack and sideslip, $\alpha=\alpha^{\mathrm{r}}, \beta=\beta^{\mathrm{r}}$, where the above conditions can be sustained. Then, the components of the aerodynamic moment are

$$
\begin{aligned}
L_{\mathrm{a}}^{\mathrm{L}}= & Q S b\left(C_{l \beta} \beta+C_{l p} \frac{p b}{2 V}+C_{l r} \frac{r b}{2 V}+C_{l \delta_{l}} \delta_{l}\right. \\
& \left.+C_{l \delta_{n}} \delta_{n}+C_{l 0}\right) \\
= & Q S b C_{l} \\
= & L_{\beta} \beta+L_{p} p+L_{r} r+L_{\delta_{l}} \delta_{l}+L_{\delta_{n}} \delta_{n}+L_{0} \\
M_{\mathrm{a}}^{\mathrm{L}}= & Q S c\left(C_{m \alpha} \alpha+C_{m q} \frac{q c}{2 V}+C_{m \delta_{m}} \delta_{m}+C_{m 0}\right) \\
= & Q S c C_{m} \\
= & M_{\alpha} \alpha+M_{q} q+M_{\delta_{m}} \delta_{m}+M_{0} \\
N_{\mathrm{a}}^{\mathrm{L}}= & Q S b\left(C_{n \beta} \beta+C_{n p} \frac{p b}{2 V}+C_{n r} \frac{r b}{2 V}+C_{n \delta_{l}} \delta_{l}\right. \\
& \left.+C_{n \delta_{n}} \delta_{n}+C_{n 0}\right) \\
= & Q S b C_{n} \\
= & N_{\beta} \beta+N_{p} p+N_{r} r+N_{\delta_{l}} \delta_{l}+N_{\delta_{n}} \delta_{n}+N_{0}
\end{aligned}
$$

where, $Q$ denotes dynamic pressure, $V$ is the intensity of air velocity vector, and $S, c, b$ are, respectively, the wing surface, mean aerodynamic chord and span. The dimensionless $C_{i j}$ and dimensional $L_{j}, M_{j}, N_{j}$ coefficients of aerodynamic moment are obtained for the moment components $i=L$, $M, N$ derived upon the variables $j=\alpha, \beta, p, q, r, \delta_{l}, \delta_{m}$, $\delta_{n}$, whereas the index $j=0$ denotes the free element in the developed sequence.

The presented method is applied as follows. The rearranged vector of angular velocity $\bar{\omega}^{\mathrm{T}}=[q \mathrm{pr}]$ is introduced, as well as the variables $\bar{\omega}^{\mathrm{T}}=[q p r] ; \quad \bar{\omega}^{\mathrm{T}}=\left[\omega_{\mathrm{L}} \omega_{\mathrm{D}}\right]$; $\omega_{\mathrm{L}}=[q] ; \quad \omega_{\mathrm{D}}^{\mathrm{T}}=[p r]$ and by substituting aerodynamic moment from Eq. (16) into Eq. (10) and rearranging, we 
get $\mathfrak{M}_{\mathrm{a}}^{\mathrm{T}} \omega=\bar{\omega}^{\mathrm{T}} \mathcal{M} \bar{\omega}+a q+b p+c r$, where $a=\left(M_{\alpha} \alpha+\right.$ $\left.M_{\delta_{m}} \delta_{m}+M_{0}\right), \quad b=\left(L_{\beta} \beta+L_{\delta_{l}} \delta_{l}+L_{\delta_{n}} \delta_{n}+L_{0}\right) \quad$ and $c=$ $\left(N_{\beta} \beta+N_{\delta_{l}} \delta_{l}+N_{\delta_{n}} \delta_{n}+N_{0}\right)$. If rearranged with the contributions of angular velocity to the aerodynamic moment separated in to longitudinal and lateral-directional parts,

$$
\begin{aligned}
\mathfrak{M}_{\mathrm{a}}^{\mathrm{T}} \omega & =\underbrace{\mathcal{M}_{\mathrm{L}} q^{2}+a q}_{\mathfrak{M}_{\mathrm{L}}^{\mathrm{T}} \omega_{\mathrm{L}}}+\underbrace{\omega_{\mathrm{D}}^{\mathrm{T}} \mathcal{M}_{\mathrm{D}} \omega_{\mathrm{D}}+b p+c r}_{\mathfrak{M}_{\mathrm{D}}^{\mathrm{T}} \omega_{\mathrm{D}}} \\
& =\mathfrak{M}_{\mathrm{L}}^{\mathrm{T}} \omega_{\mathrm{L}}+\mathfrak{M}_{\mathrm{D}}^{\mathrm{T}} \omega_{\mathrm{D}} \\
\mathfrak{M}_{\mathrm{L}}^{\mathrm{T}} \omega_{\mathrm{L}} & =\mathcal{M}_{\mathrm{L}} q^{2}+a q ; \\
\mathfrak{M}_{\mathrm{D}}^{\mathrm{T}} \omega_{\mathrm{D}} & =\omega_{\mathrm{D}}^{\mathrm{T}} \mathcal{M}_{\mathrm{D}} \omega_{\mathrm{D}}+[b c] \omega_{\mathrm{D}} ; \\
\mathcal{M}_{\mathrm{D}} & =\left[\begin{array}{ll}
L_{p} & L_{r} \\
N_{p} & N_{r}
\end{array}\right]
\end{aligned}
$$

where, $\omega_{\mathrm{L}}$ is the longitudinal part and $\omega_{\mathrm{D}}$ is the lateral-directional part of the angular velocity vector, $\mathcal{M}$ is the matrix of contributions of angular velocity to the aerodynamic moment, while $\mathcal{M}_{\mathrm{L}}=\left[M_{q}\right]$ and $\mathcal{M}_{\mathrm{D}}$ are its longitudinal and lateral-directional parts. Note, that separation is performed only on longitudinal and lateral-directional parts of aerodynamic moment, whereas the analysis of airplane rotation dynamics remained integral.

Geometrical interpretation of Eq. (17) is simple. Equation (17) is observed in the four-dimensional space $O \dot{E}_{\mathrm{R}} q p r$, with the axes $O \dot{E}_{\mathrm{R}}, O q, O p$ and $O r$. Note that $\mathrm{d} / \mathrm{d} t\left(E_{\mathrm{R}}\right)=\mathfrak{M}_{\mathrm{a}}^{\mathrm{T}} \omega$. Selection of $O \dot{E}_{\mathrm{R}}$ instead of $\mathfrak{M}_{\mathrm{a}}^{\mathrm{T}} \omega$ for designating the axis in Eq. (17) is done to emphasize its physical meaning. The variable $O \dot{E}_{\mathrm{R}}$ represents the sum of the hyperparaboloid $\bar{\omega}^{\mathrm{T}} \mathcal{M} \bar{\omega}$ and plane $a q+b p+c r$ containing the point of the coordinate frame origin $O$. The plane can rotate about the point $O$ with the position determined by the values of coefficients $a, b$ and $c$, which are dependent on the instantaneous values of state variables $\alpha, \beta$ and deflection of the control surfaces $\delta_{l}, \delta_{m}$ and $\delta_{n}$. In the case of a stationary, straight, wing-level flight of a geometrically symmetric airplane, geometrical interpretation of Eq. (17) reduces to the coordinate hyper-plane $O q p r$.

In analysis of airplane rotation dynamics, the transfer from the general form of condition in Eq. (10) to the form in Eq. (17) is based on a locally linearized model of airplane aerodynamic moments. Stationary states of airplane dynamics represent equilibrium states from the flight reference point. The equilibrium states of flight are only a subset in the range of angles of attack and sideslip, $\alpha, \beta$, where local linearization can be performed.

\section{Relationship of Classical Approach to Analysis of Airplane Rotation Dynamics}

A special case of flight equilibrium is the stationary, straight, horizontal, wing-level flight. For an inertially and geometrically symmetric airplane, these are the conditions for the classical approach to analysis of airplane dynamics, based on separated modes of longitudinal and lateral-directional motion. Let us denote the equilibrium flight states by the superscript ' $e$ ', so that for the symmetric airplane case coefficients $a, b$ and $c$ in Eq. (17) are zero as

$$
\begin{aligned}
& a^{\mathrm{e}}=M_{\alpha} \alpha^{\mathrm{e}}+M_{\delta_{m}} \delta_{m}^{\mathrm{e}}+M_{0}^{\mathrm{e}}=0 \\
& b^{\mathrm{e}}=L_{\beta} \beta^{\mathrm{e}}+L_{\delta_{l}} \delta_{l}^{\mathrm{e}}+L_{\delta_{n}} \delta_{n}^{\mathrm{e}}+L_{0}^{\mathrm{e}}=0 \\
& c^{\mathrm{e}}=N_{\beta} \beta^{\mathrm{e}}+N_{\delta_{l}} \delta_{l}^{\mathrm{e}}+N_{\delta_{n}} \delta_{n}^{\mathrm{e}}+N_{0}^{\mathrm{e}}=0 .
\end{aligned}
$$

Then, the values of stream angles and control surface deflections are $\alpha^{\mathrm{e}} \neq 0, \delta_{m}^{\mathrm{e}} \neq 0, \beta^{\mathrm{e}}=0, \delta_{l}^{\mathrm{e}}=0$ and $\delta_{n}^{\mathrm{e}}=0$, as well as $L_{0}^{\mathrm{e}}=0$ and $N_{0}^{\mathrm{e}}=0$, because the last two equations must be identically satisfied. In the case of a non-symmetric airplane, $\beta^{\mathrm{e}} \neq 0, \delta_{l}^{\mathrm{e}} \neq 0$ and $\delta_{n}^{\mathrm{e}} \neq 0, L_{0}^{\mathrm{e}} \neq 0$ and $N_{0}^{\mathrm{e}} \neq 0$, so the analysis reverts to the locally linearized model (Eq. (17)).

To demonstrate the congruency of the analysis of airplane rotational dynamics based on the condition in Eq. (10) with the classical approach to this problem, it is necessary to subject it to the same set of limitations and constraints that have been used in developing decomposed linearized models of longitudinal and lateral-directional modes of airplane motion. A brief review of these constraints follows. Models are derived as linear for the above initial conditions of equilibrium state of a symmetric airplane. The range of values of airplane state variables is limited to the domain of their linear functional dependability, the most important being the range of angle of attack of up to $\alpha \leq 0.8 \alpha_{\text {crit }}$, where $\alpha_{\text {crit }}$ is its critical value. For a defined set of stream parameters, Mach $M$ and Reynolds Re numbers, the dimensionless $C_{i j}$ and dimensional $L_{j}, M_{j}, N_{j}$ coefficients of aerodynamic moment are constant within this range of linearity.

Stability analysis within each of the separated modes is limited to the domain of validity of a particular linear model. An overall stability of the airplane exists if and only if there is simultaneous local stability in each of the particular modes of airplane motion.

The meaning of these constraints applied to the condition in Eq. (10), modified to the form in Eq. (17) is as follows. The expression $\mathfrak{M}_{\mathrm{a}}^{\mathrm{T}} \omega$ attains sign definiteness if and only if the overall expression elements $\mathfrak{M}_{\mathrm{L}}^{\mathrm{T}} \omega_{\mathrm{L}}$ and $\mathfrak{M}_{\mathrm{D}}^{\mathrm{T}} \omega_{\mathrm{D}}$ attain sign definiteness, independently and simultaneously. For example, a non-positive sign definiteness $\mathfrak{M}_{\mathrm{a}}^{\mathrm{T}} \omega \leq 0$ can be attained if and only if, simultaneously and independently, $\mathfrak{M}_{\mathrm{L}}^{\mathrm{T}} \omega_{\mathrm{L}} \leq 0$ and $\mathfrak{M}_{\mathrm{D}}^{\mathrm{T}} \omega_{\mathrm{D}} \leq 0$. The application of constraints in the classical approach allows each of these elements, $\mathfrak{M}_{\mathrm{L}}^{\mathrm{T}} \omega_{\mathrm{L}}$ and $\mathfrak{M}_{\mathrm{D}}^{\mathrm{T}} \omega_{\mathrm{D}}$, of the integral relation Eq. (17) to be considered separately.

\subsection{Application of method to decoupled longitudinal motion}

To apply the condition of Eq. (10) to the analysis of a linear model of the longitudinal mode of airplane motion it suffices to observe only the expression

$$
\begin{aligned}
\frac{\mathrm{d}\left(E_{q}\right)}{\mathrm{d} t}= & \mathfrak{M}_{\mathrm{L}}^{\mathrm{T}} w_{\mathrm{L}} \\
& =\mathcal{M}_{\mathrm{L}} q^{2}+a q \\
& =M_{q} q^{2}+\left(M_{\alpha} \alpha+M_{\delta_{m}} \delta_{m}+M_{0}\right) q \leq 0, \\
\dot{E}_{q} & =\mathfrak{M}_{\mathrm{L}}^{\mathrm{T}} w_{\mathrm{L}} \\
& =M_{q}\left[\left(q+\frac{a}{2 M_{q}}\right)^{2}-\left(\frac{a}{2 M_{q}}\right)^{2}\right] \leq 0, \\
& \quad M_{q} \neq 0 .
\end{aligned}
$$




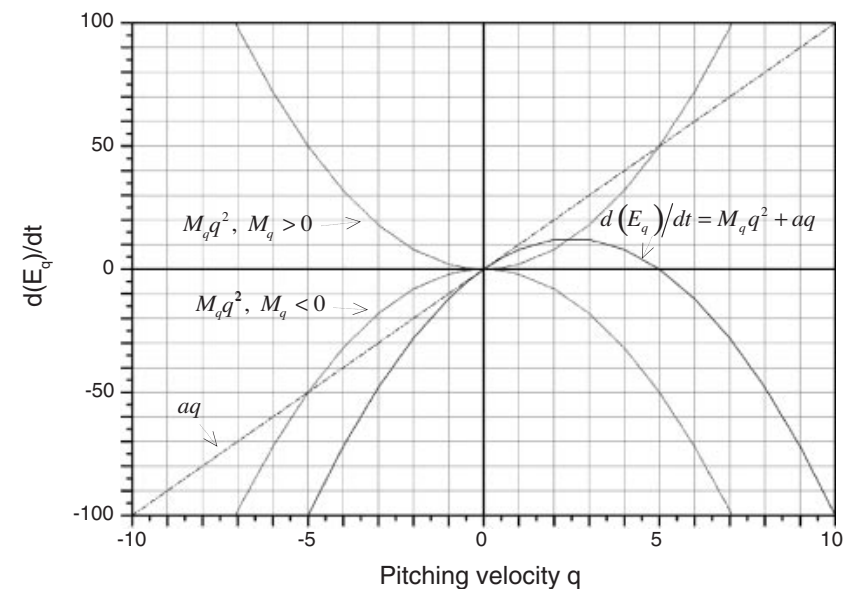

Fig. 2. Rate of energy of rotation in longitudinal plane.

In Eq. (19) $E_{q}$ is the separated kinetic energy of pitching (longitudinal) angular motion. The second form of condition in Eq. (19) points out the shape and elements of a parabola. The significance of the simplification made in Eq. (19) can be best understood if a comparison is made with the inherent form of kinetic energy of rotation presented as the ellipsoid of rotation in Eq. (3). By assumption, separated observation of the airplane longitudinal motion mode reduces rolling and yawing component of the angular velocity to zero, i.e. $p=0$ and $r=0$, which, by analogy with the ellipsoidal rigid body, is equivalent to its reduction to a thin stick. Therefore, the variation of rotational energy is generated only along the fixed direction $O q$.

Geometrical interpretation of the condition in Eq. (19) is carried out in the two-dimensional space $O \dot{E}_{q} q$, with the axes $O \dot{E}_{q}$ and $O q$ (Fig. 2). The function $\mathfrak{M}_{\mathrm{L}}^{\mathrm{T}} \omega_{\mathrm{L}}=$ $M_{q} q^{2}+a q$ is a sum of the parabola $M_{q} q^{2}$ and the straight line $a q$ that contains the coordinate origin $O$, and is defined for any value of $M_{q}$. The second form of condition in Eq. (19) determines the extreme of the parabola and is defined only when $M_{q} \neq 0$. The condition $M_{q}=0$ is extremely rare and practically impossible for airplanes with empennage. Angular orientation of the straight line is defined by the coefficient $a$. The direction coefficient in Eq. (18) is $a^{\mathrm{e}}=0$ for the airplane in equilibrium flight, and the straight line $a q$ is collinear with the axis $O q$. For a decomposed motion, $M_{0}^{\mathrm{e}}=\left.M_{0}\right|_{\alpha=0}$, because $\mathfrak{M}_{\mathrm{L}}(\alpha)$ is linear.

For the above equilibrium initial state, the necessary condition for a stable rotation (pitching) of the airplane in the separated longitudinal mode is $\mathfrak{M}_{\mathrm{L}}^{\mathrm{T}} \omega_{\mathrm{L}}=M_{q} q^{2}<0$, from where requirement $M_{q}<0$ emerges. The boundary stability condition is $M_{q} q^{2}=0$, while for unstable pitching motion, it is $M_{q} q^{2}>0$. Any deviation from the equilibrium state produces the increment $\Delta a$ of the coefficient $a$, so that $a=a^{\mathrm{e}}+\Delta a \neq 0$, and the straight line is $\Delta a q$, i.e. rotated by $\Delta a$. The parabola extreme is then shifted from the coordinate origin along the $O q$ axis by $\left(-\Delta a / 2 M_{q}\right)$. Then, the parabola representing the condition in Eq. (19) has two zeroes and two sets of values with opposite signs.

For example, for a stable airplane and the above equilibrium, the initial state is $M_{q}<0$ and the element $M_{q} q^{2}$ is with non-positive values. Deviation from the equilibrium causes the sum $M_{q} q^{2}+\Delta a q$ to have region of positive values. The deviation can be due to the required deflection of the control surface $\Delta \delta_{m}$ or to environment disturbance in the form of either $\Delta \alpha$ or variations of the coefficients $\Delta M_{\alpha}, \Delta M_{\delta_{m}}$ and $\Delta M_{0}$.

To prevent increase of rotation in the longitudinal mode of motion for the known amount of rotation $R$, the boundary condition $\mathfrak{M}_{\mathrm{L}}^{\mathrm{T}} \omega_{\mathrm{L}}=M_{q} q^{2}+a q=0$ must be fulfilled for the value of pitching angular velocity $q= \pm R / \sqrt{J_{2}}$. In the domain of constant aerodynamic parameters, the necessary boundary increment of control surface deflection is determined from the condition $M_{q}\left(R / \sqrt{J_{2}}\right)+M_{\alpha}\left(\alpha^{\mathrm{e}}+\Delta \alpha\right)+$ $M_{\delta_{m}}\left(\delta_{m}^{\mathrm{e}}+\Delta \delta_{m}\right)+M_{0}^{\mathrm{e}}=0$. Consequently, the boundary increment of control surface deflection needed to prevent increase of rotation in the longitudinal mode of airplane motion is $\left(\Delta \delta_{m}\right)_{b}=\left(M_{q}\left(R / \sqrt{J_{2}}\right)-M_{\alpha} \Delta \alpha\right) / M_{\delta_{m}}$, which is in full agreement with the requirement given by Etkin. ${ }^{2)}$

The condition of stability of longitudinal motion stems from the demand to fulfill the requirement $\mathfrak{M}_{\mathrm{L}}^{\mathrm{T}} \omega_{\mathrm{L}}<0$ in the presence of the disturbance $\Delta \alpha$ relative to the existing equilibrium state (Eq. (22)). As the necessary condition is $M_{q} q^{2}<0$, the sufficient condition $\mathfrak{M}_{\mathrm{L}}^{\mathrm{T}} \omega_{\mathrm{L}}=\left(M_{\alpha} \Delta \alpha / q+\right.$ $\left.M_{q}\right) q^{2}<0$ for $q \neq 0$ emerges. As $M_{\alpha}=Q S c C_{m \alpha}, M_{q}=$ $Q S c C_{m q}(c / 2 V)$ and $q / \Delta \alpha=-Z_{\alpha} / m V=-C_{z \alpha}(Q S / m V)$, the previous condition becomes $C_{m q} C_{z \alpha} \cdot(c / 2 V)-$ $C_{m \alpha}(m V / Q S)>0$, which is identical to the condition for longitudinal motion stability given by Blakelock. ${ }^{1)}$

The previous discussion shows that classical approaches to analysis of airplane longitudinal rotation are particular solutions to the general condition in Eq. (10).

\subsection{Application of method to decoupled lateral motion}

To analyze the lateral-directional mode of airplane motion on the basis of Eq. (10), it suffices to observe separately the expression

$$
\begin{aligned}
\frac{\mathrm{d}\left(E_{\mathrm{D}}\right)}{\mathrm{d} t} & =\dot{E}_{\mathrm{D}}=\mathfrak{M}_{\mathrm{D}}^{\mathrm{T}} \omega_{\mathrm{D}} \\
& =\omega_{\mathrm{D}}^{\mathrm{T}} \mathcal{M}_{\mathrm{D}} \omega_{\mathrm{D}}+b p+c r \leq 0,
\end{aligned}
$$

where, $E_{\mathrm{D}}$ is the separated kinetic energy of lateraldirectional angular motion and $\mathcal{M}_{\mathrm{D}}$ and $\omega_{\mathrm{D}}$ are of the same forms as in the case of locally linearized model of aerodynamic moment. By assumption, the separated observation of lateral-directional mode of airplane motion reduces to zero pitching angular velocity, i.e. $q=0$. Then, the inherent form of kinetic energy of rotation presented as the ellipsoid of rotation in Eq. (3) is reduced to the ellipse in the Opr plane

$$
2 E_{\mathrm{D}}=J_{1} p_{J}^{2}+J_{3} r_{J}^{2}=R_{\mathrm{D}}^{2} .
$$

Geometrical interpretation of the condition in Eq. (20) is given in the $3 \mathrm{D}$ space $O \dot{E}_{\mathrm{D}} p r$, with the axes $O \dot{E}_{\mathrm{D}}, O p$ and Or. The function $\omega_{\mathrm{D}}^{\mathrm{T}} \mathcal{M}_{\mathrm{D}} \omega_{\mathrm{D}}+b p+c r$ represents a sum of the paraboloid $\omega_{\mathrm{D}}^{\mathrm{T}} \mathcal{M}_{\mathrm{D}} \omega_{\mathrm{D}}$ and the plane $b p+c r$ containing the coordinate origin $O$. The plane $b p+c r$ can rotate about the coordinate origin $O$, with the orientation defined by the coefficients $b$ and $c$, i.e. by the straight lines $b p$ in the coor- 
dinate plane $O \dot{E}_{\mathrm{D}} p$ and $c r$ in the coordinate plane $O \dot{E}_{\mathrm{D}} r$. The intersection of the paraboloid $\omega_{\mathrm{D}}^{\mathrm{T}} \mathcal{M}_{\mathrm{D}} \omega_{\mathrm{D}}$ and the plane $\dot{E}_{\mathrm{D}}=$ constant parallel to the coordinate plane $O p r$ is an ellipse with the main axes parallel to the first and third main axes of inertia of the airplane as a rigid body.

For the airplane in equilibrium flight, from Eq. (18) $b^{\mathrm{e}}=$ 0 and $c^{\mathrm{e}}=0$, then the plane $b p+c r$ and coordinate plane $O p r$ are coplanar. For the above equilibrium initial state, the necessary condition for a stable rotation of airplane in the separated lateral-directional mode is $\mathfrak{M}_{\mathrm{D}}^{\mathrm{T}} \omega_{\mathrm{D}}=$ $\omega_{\mathrm{D}}^{\mathrm{T}} \mathcal{M}_{\mathrm{D}} \omega_{\mathrm{D}}<0$, from where emerges the requirement $\mathcal{M}_{\mathrm{D}}<$ 0 for a negative sign definiteness of the matrix $\mathcal{M}_{\mathrm{D}}$. According to Bellman, ${ }^{9)}$ this requirement is satisfied if all main minors of this matrix are negative. Expressed through the dimensionless and dimensional coefficients, it is $L_{p}=\left(Q S b^{2} / 2 V\right) C_{l p}<0, \quad C_{l p}<0 \quad$ and $\quad L_{p} N_{r}-N_{p} L_{r}=$ $\left(Q S b^{2} / 2 V\right)\left(C_{l p} C_{n r}-C_{n p} C_{l r}\right)<0$. The same requirement has also been defined elsewhere. ${ }^{1,2,10)}$ The condition for boundary stability is when $\mathcal{M}_{\mathrm{D}}=0$ and is instability when $\mathcal{M}_{\mathrm{D}}>0$.

Any deviation from the equilibrium state produces the increments $\Delta b$ and $\Delta c$, that is $b=b^{\mathrm{e}}+\Delta b \neq 0$ and $c=$ $c^{\mathrm{e}}+\Delta c \neq 0$. The plane equation becomes $\Delta b p+\Delta c r$, and the plane rotates about the coordinate origin $O$ by the angle $\Delta b$ in the coordinate plane $O \dot{E}_{\mathrm{D}} p$ and by the angle $\Delta c$ in the coordinate plane $O \dot{E}_{\mathrm{D}} r$. Then, the paraboloid apex is shifted from the coordinate origin, generating three regions of condition Eq. (20). The first contains zero values of the condition in Eq. (20), representing an ellipse in the coordinate plane $\mathrm{Opr}$, with the other two representing surfaces of opposite signs.

For example, for a stable airplane and the above equilibrium initial state, $\mathcal{M}_{\mathrm{D}}<0$, the element $\omega_{\mathrm{D}}^{\mathrm{T}} \mathcal{M}_{\mathrm{D}} \omega_{\mathrm{D}}$ has a nonpositive value. Deviation from equilibrium causes the sum $\omega_{\mathrm{D}}^{\mathrm{T}} \mathcal{M}_{\mathrm{D}} \omega_{\mathrm{D}}+\Delta b p+\Delta c r$ to have a region of positive values. The deviation can be a consequence of the required deflection of control surfaces $\Delta \delta_{l}$ and $\Delta \delta_{n}$ or by environment disturbance in the form of either $\Delta \beta$ or variations of the coefficients $\Delta L_{\beta}, \Delta L_{\delta_{l}}, \Delta L_{\delta_{n}}, \Delta L_{0}, \Delta N_{\beta}, \Delta N_{\delta_{l}}, \Delta N_{\delta_{n}}$ and $\Delta N_{0}$.

The condition of stability of the longitudinal motion stems from the demand to fulfill the requirement $\mathfrak{M}_{\mathrm{D}}^{\mathrm{T}} \omega_{\mathrm{D}}<0$ in the presence of the disturbance $\Delta \beta$ relative to the existing equilibrium state (Eq. (18)), so that $\beta^{\mathrm{e}}=0$ and $\beta=\Delta \beta$. A necessary condition is always $\mathcal{M}_{\mathrm{D}}<0$, so that a sufficient condition for stability of the airplane in the lateral-directional motion in the presence of the disturbance $\Delta \beta$ is that the angular velocities $p$ and $r$ are such that the requirement $b p+c r<0$ is fulfilled. The boundaries of the area where this inequality is valid can be determined from the expression

$$
\begin{aligned}
b p+c r= & \left(L_{\beta} \beta+L_{\delta_{l}} \delta_{l}+L_{\delta_{n}} \delta_{n}\right) p \\
& +\left(N_{\beta} \beta+N_{\delta_{l}} \delta_{l}+N_{\delta_{n}} \delta_{n}\right) r=0,
\end{aligned}
$$

because $L_{0}^{\mathrm{e}}=L_{0}=0$ and $N_{0}^{\mathrm{e}}=N_{0}=0$. One partial solution of this equation is its identical equality when $b=0$ and when $c=0$. Computing $\beta$ from $b=0$ and substituting into $c=0$ yields the condition

$$
N_{\beta}-L_{\beta} \frac{N_{\delta_{l}}+N_{\delta_{n}} \frac{\delta_{l}}{\delta_{n}}}{L_{\delta_{l}}+L_{\delta_{n}} \frac{\delta_{l}}{\delta_{n}}}=0,
$$

which defines relations in the stationary turning flight with sideslip.

From the same boundary condition, an equivalent conclusion can be drawn. $\delta_{l} / \beta$ from $b=0$ and substituting into $c=0$ yields

$$
N_{\beta}-L_{\beta} \frac{N_{\delta_{l}}}{L_{\delta_{l}}}\left(L_{\delta_{n}} \frac{N_{\delta_{l}}}{L_{\delta_{l}}}-N_{\delta_{n}}\right)\left(\frac{\delta_{n}}{\beta}\right)=0 .
$$

For an undeflected rudder, $\delta_{n}=0$, the previous equation reduces to

$$
N_{\beta} L_{\delta_{l}}-L_{\beta} N_{\delta_{l}}=0
$$

If in the condition of Eq. (20) we take only the elements multiplied by $r$ and $\beta p$, where $p=$ constant, the stability condition is $y=L_{\beta} \beta p+N_{\beta} \beta r+L_{r} r p+N_{r} r^{2}<0$. The function $y$ is a parabola with the yawing angular velocity $r$ as an independent variable. The value of independent variable $r_{a x}=-\left(N_{\beta} \beta+L_{r} p /\left(2 N_{r}\right)\right)$ yields the apex of the parabola $y$ as

$$
y_{a x}=\frac{2\left(L_{\beta} N_{r}+N_{\beta} L_{r}\right) \beta p+N_{\beta}^{2} \beta^{2}+L_{r}^{2} p^{2}}{2 N_{r}} .
$$

For an airplane with empennage, $N_{r}$ is negative in the area of subcritical angles of attack, therefore the apex of $y$ is its maximum. Generally, $y$ has two zeroes and two sets of values of opposite signs. If the function $y$ must be negative, then $y_{a x}<0$. One of the boundaries is

$$
L_{\beta} N_{r}+N_{\beta} L_{r}=0,
$$

as the sum of the squares in the condition of Eq. (25) is always positive. Note that all obtained conditions are partial solutions of the general condition $\mathfrak{M}_{\mathrm{D}}^{\mathrm{T}} \omega_{\mathrm{D}}<0$, and are fully congruent with the previously reported results. ${ }^{1,11,12)}$

This section shaws the full congruency of partial solutions of the condition given in Eq. (10) and results obtained from the classical approach to the analysis of airplane lateraldirectional rotation.

Note that analyse in the literature give conditions for airplane rotation related to the aerodynamic moments linearized as the functions of state variables. Application of this method can give new conditions that simultaneously combine airplane state variables and inertial properties. ${ }^{13)}$

\section{Conclusion}

This new methodology to analyze the dynamic behavior of a rotating airplane in the whole range of angles of attack is based on the fact that the character of rotational motion of an airplane as a rigid body is determined by the rate of kinetic energy of rotation. This quantity is equal to the derivative of energy of rotation along the vector of angular velocity. By applying the principles of classical mechanics 
and using a full non-linear model of airplane motion, we prove that it represents a scalar product of the vector of moment and vector of angular velocity of the airplane. The exactness depends only upon the exactness of the source of aerodynamic and propulsive moments applied in it, providing exact results for exact functions of input moments.

The concept of a model of locally linearized aerodynamic moment is introduced with local applicability throughout the whole range of angles of attack. Application of this model enables simultaneous separate observation of longitudinal and lateral-directional motion, because the linearization is performed only upon the aerodynamic moments acting on the airplane, and not upon presentation of the motion itself.

Within the stringent constraints of the classical approach based on the separated modes of longitudinal and lateraldirectional motion, there is full congruency of partial results of this method and the results of classical methods. On the other hand, the general applicability of this method has also been demonstrated in areas where classical approaches fail to produce valid results. The first domain of application is analysis of lateral-directional rotation generated by longitudinal command. The second domain is concerned with definition of conditions needed to prevent increase and/or stoppage of the lateral-directional rotation of the airplane, so these conditions are applicable within the whole range of interest of the values of the angles of attack. The results in the second domain yield some new conditions and relations between the values of aerodynamic and inertial parameters of an airplane on one hand, and intensity of airplane rotation on the other. To apply the method is sufficient to know algebra and the functional relations of aerodynamic moments (values of aerodynamic parameters) throughout the whole range of flight regimes.

\section{References}

1) Blakelock, J.: Automatic Control of Aircraft and Missiles, John Wiley \& Sons, New York, 1991.

2) Etkin, B.: Dynamics of Atmospheric Flight, John Wiley \& Sons, New York, 1972.

3) Beaurain, L.: Influence of Light Aircraft Architecture on Its Spin Characteristics, Tech. Rep. AAAF-NT-80-11, Institute de Mechanique de Fluides de Lille, Lille, 1979.

4) Kaus, W.: Aerodynamic Development of the $x-31$ for the High Angle of Attack Region, Tech. Rep. MBB-FE211-S-PUB-452; ETN-92-90620, Messerschmidt-Boelkow-Blohm G.m.b.H., Munich, 1991.

5) Mehre, R. and Carroll, J.: Global Stability and Control Analysis of Aircraft at High Angles-of-attack, Tech. Rep. ONR-cr 215-245-3, Scientific Systems Inc., Cambridge, MA, USA, 1979.

6) Tobak, M. and Chapman, G.: Nonlinear Problems in Flight Dynamics Involving Aerodynamic Bifurcation, Tech. Rep. NASA-TM-86706, NASA, Ames Research Center, Moffet Field, CA, USA, 1981.

7) Hill, S. and Martin, C.: Flight Dynamics Model of Aircraft Spinning, ARL-FLIGHT-MECH-R-180; DODA-AR-005-600, Aeronautical Research Labs, Melbourne, 1990.

8) Stiefel, J.: Math Model for Nadc Spin Simulator and f-14 High Angle of Attack Data Base, Tech. Rep. NADC-80220-60-VOL-1, Naval Air Development Center, Aircraft and Crew Systems Technology Directorate, Earminster, PA, USA, 1981.

9) Bellman, R.: Introduction to Matrix Analysis, McGraw-Hill Inc., New York, 1960.

10) Iliff, K. and Maine, R.: Application of Parameter Estimation to Aircraft Stability and Control: The Output Error Approach, Tech. Rep. NASA-RP-1186, 1986.

11) Bajovic, M.: Airplane Motion Analysis at the High Angles-of-attack, Tech. Rep. V3-1980, Aeronautical Institute, Belgrade, Serbia, 1981.

12) Bajovic, M.: New Approach in the Solution of Aircraft Flight Dynamics Problem from the Flying Qualities Point of View, PhD Thesis, Faculty of Mechanical Engineering, University of Belgrade, 2002.

13) Zivanovic, M., Bajovic, M., Stojakovic, P. and Rasuo, B.: New General Approach to the Airplane Rotation Analysis, ICAS 2004, Yokohama, Japan, 2004. 